\title{
BMJ Open Interventions to improve the self-management support health professionals provide for people with progressive neurological conditions: protocol for a realist synthesis
}

\author{
Freya Davies, ${ }^{1}$ Fiona Wood, ${ }^{1}$ Alison Bullock, ${ }^{2}$ Carolyn Wallace, ${ }^{3}$ Adrian Edwards ${ }^{1}$
}

To cite: Davies F, Wood F, Bullock A, et al. Interventions to improve the self-management support health professionals provide for people with progressive neurological conditions: protocol for a realist synthesis. BMJ Open 2017;7: e014575. doi:10.1136/ bmjopen-2016-014575

- Prepublication history and additional material is available. To view please visit the journal (http://dx.doi.org/ 10.1136/bmjopen-2016014575).

Received 4 October 2016 Revised 29 November 2016 Accepted 12 January 2017

\section{CrossMark}

\author{
${ }^{1}$ Division of Population \\ Medicine, Cardiff University, \\ Cardiff, UK \\ ${ }^{2}$ Cardiff Unit for Research \\ and Evaluation in Medical \\ and Dental Education, Cardiff \\ University, Cardiff, UK \\ ${ }^{3}$ Faculty of Life Sciences and \\ Education, University of \\ South Wales, Pontypridd, UK
}

Correspondence to

Dr Freya Davies;

daviesf9@cardiff.ac.uk

\section{ABSTRACT}

Introduction: Supporting self-management among people with long-term conditions is recognised as an important component of healthcare. Progressive neurological conditions (PNCs), for example, Parkinson's disease and multiple sclerosis are associated with problems such as fatigue and cognitive impairment which may make self-management more challenging. Health professionals may need to develop specific skills in order to provide effective selfmanagement support for these patients. The review aims to develop explanatory theories about how health professional-targeted interventions to improve selfmanagement support provision for people with PNCs operate in different circumstances.

Methods and analysis: A realist synthesis of the evidence is proposed. There are 2 priority questions for the review to address. These relate to the role of a shared concept of self-management support within the healthcare team, and the need to tailor the support provided to the requirements of people with PNCs. Key stakeholders will be involved throughout the process. The initial search strategy uses terms relating to (1) self-management, (2) health professionals and (3) PNCs. Searching, data extraction and synthesis will occur in parallel. Studies will be prioritised for inclusion based on anticipated contribution to generating explanatory theories. Key informant interviews are planned to direct supplementary searches and help further refine the theories developed. Results will be expressed in the form of context-mechanism-outcome configurations.

Ethics and dissemination: Publication guidelines on realist synthesis will be followed. The results will be published in a peer-reviewed journal and made available to organisations involved in the provision of health professional training.

\section{INTRODUCTION}

People living with long-term conditions make decisions that relate to the management of

\section{Strengths and limitations of this study}

- The application of a realist approach to evidence synthesis will lead to theory development about the contexts in which interventions are most likely to succeed.

- Describing the mechanisms by which existing interventions work will facilitate future theorydriven intervention design and evaluation.

- The breadth of interventions which might be considered to support self-management may make defining the scope of the review challenging.

- If evidence available relating to supporting people with progressive neurological conditions is limited the reviewers will need to consider the transferability of knowledge generated in other settings.

their condition on a daily basis, ${ }^{1}$ from choosing how they use their medication to how they plan their activities. Corbin and Strauss ${ }^{2}$ suggest that self-managing a condition involves three tasks: medical management, role management and emotional management. Health professionals have tended to focus on optimising the medical management of conditions, but there is increasing understanding that the focus of efforts may need to shift towards an approach that encompasses all of these tasks to help people to live well with their condition. ${ }^{3}$ People often have different definitions of successful self-management compared with their clinicians, with patients emphasising the need for self-management support (SMS) that is relevant to the context of their lives. ${ }^{4}$ This may be particularly important in progressive neurological conditions (PNCs). PNCs are conditions in which patients experience a progressive deterioration in their functioning 
(eg, Parkinson's disease and multiple sclerosis). In these conditions successful self-management is not necessarily expected to modify the disease course itself, but may have a significant impact on how well people live with their symptoms.

\section{What is SMS?}

SMS may be delivered directly to patients, for example, via attendance at SMS programmes. ${ }^{5}$ These programmes may include activities such as information provision, emotional and behavioural management skills, and technical skill development. ${ }^{6} \quad 7$ Condition-specific selfmanagement programmes for people with a PNC often cover issues such as physical activity, medication adherence, cognitive impairment, depression and fatigue. ${ }^{8}$ Limitations to these types of approaches have been recognised, including the fact that patients who volunteer to attend such programmes may already be motivated and skilled in self-management, ${ }^{9}$ while those who may benefit from support most may not access these types of courses. ${ }^{10}$ If SMS becomes integrated into routine clinical care more patients will have access to support. To encourage this integration, interventions aiming to promote SMS may include indirect components delivered either to individual professionals (such as education and training) or at an organisational level (eg, financial incentives). ${ }^{11}$ The variety of skills health professionals require to effectively support selfmanagement has been broadly divided into general person-centred skills (such as communication skills), behaviour change skills (eg, motivational interviewing) and organisation/system skills (eg, use of electronic recall systems). ${ }^{12}$

\section{What is known about training health professionals to support self-management?}

Supporting self-management is not a straightforward task for clinicians as it requires judgements to be made around patient readiness, professional role boundaries and service expectations. The evidence for training health professionals to support self-management is mixed. While there is some evidence that training health professionals can change clinicians' behaviours, ${ }^{13}$ others have shown that clinicians failed to apply training in SMS in their routine work. ${ }^{14}$ Implementation of SMS in routine practice is recognised to be inherently complex, with multiple potential barriers at the levels of the patient, the professional and the wider organisation. ${ }^{11}$ The need for further research to understand how provider burden can be minimised and selfmanagement programmes can be made more widely acceptable has been recognised. ${ }^{15}$

Previous suggestions for optimising professionaltargeted interventions include involving staff members in the intervention design process; and ensuring that any intervention is seen as professionally desirable, and fits within existing clinical routines. ${ }^{11} 16$ The context into which an intervention will be delivered should be considered if the intervention design is to be successful. In particular, staff preconceptions about their role in supporting self-management, and its relative importance in relation to other tasks should be addressed. ${ }^{14}$

\section{Supporting self-management in the context of PNCs}

Although supporting self-management has been shown to be challenging across a range of settings, supporting people with a PNC may raise particular issues. Depression, cognitive impairment and fatigue are common comorbidities in PNCs and may all make it more challenging for patients to effectively self-manage, and for professionals to know how best to support selfmanagement in these circumstances. ${ }^{17}$ Professionals working in this area already have a wide remit including providing education and support, symptom management, medication advice, care coordination, and ongoing care planning. High workloads and a lack of time to meet all patient needs have been reported. ${ }^{18}$ Much of the available research evidence relating to selfmanagement comes from conditions, such as diabetes, where objective measures of disease control which may respond to successful self-management are available. In PNCs the expected outcomes of supporting selfmanagement are likely to be harder for professionals to define and measure. While this may mean that professionals are encouraged to take a more holistic view of supporting self-management than a narrow focus on the medical management of a condition, it may also lead to difficulties in recognising how interventions to support self-management add value to routine clinical care.

Professionals are required to make their own judgements about the level of self-management that they might expect their patients to engage in, and the level of support they provide to attempt to facilitate this process. Interventions aiming to improve SMS provision need to influence these decisions. For example, some professionals may worry that expecting people with a PNC to take an active role in self-managing their condition could be excessively burdensome. Training which encourages exploration of the purpose and goals of SMS may work well for this staff group. Others may feel that they lack the time required to provide SMS. In this case, training which provides ideas which can be easily integrated into their current practice may be seen as most valuable. A review approach designed to take into account this type of complexity is therefore required.

\section{METHODS AND ANALYSIS \\ Chosen methodology}

Research into continuing professional development activities has been criticised for focusing only on whether or not interventions work-without attention being paid to the mechanisms by which they have an effect $^{19}$ or the relevant contextual influences that moderate their effectiveness. The realist synthesis approach has been proposed as an effective method for 
synthesising evidence from complex interventions which addresses these concerns. ${ }^{20} \mathrm{~A}$ realist synthesis uses a theory-driven approach, informed by an acknowledgement that interventions will operate differently when delivered into different contexts. Realist synthesis seeks to unpick what type of intervention works, for which professionals, working in which settings, to what extent and why. This is done through the development of programme theories, developed and refined throughout the review process, which describe how the context into which the intervention is delivered influences how the intervention functions (its 'mechanism') in order to produce a range of differing intended and unintended outcomes.

We plan to use the realist synthesis approach to review the evidence about interventions which aim to increase or improve the support for self-management provided by health professionals working with people with PNCs. Training health professionals in SMS is by definition a complex intervention, consisting of multiple interacting components $^{21}$ and therefore well suited to a synthesis approach that acknowledges this complexity. During this review, we will focus specifically on understanding how training in SMS and delivery of support operates at the level of the health professional, rather than at the level of the patient.

The approach to searching for evidence in a realist review is more iterative than a traditional review procedure, and allows reviewers to purposively search for and select literature likely to be informative. ${ }^{20}$ This is likely to be particularly helpful in this review because selfmanagement itself is a complex concept to define, and this also makes a conventional literature search challenging. ${ }^{22}$ The more inclusive nature of a realist review allows data which may not be indexed under the heading of SMS (but do relate to an important element of SMS) to be included, as researcher judgement on relevance is used in place of strict inclusion/exclusion criteria.

Realist reviews operate at the level of transferable programme theories (rather than at the level of a specific intervention). As a result, realist reviewers recognise the transferability of knowledge from other settings and may include evidence from areas that relate to the programme theory (but not necessarily the narrow topic area under review). Again this is likely to be relevant here, to ensure that this review identifies sufficient evidence to be useful and informative. A recent review of systematic reviews of self-management identified only limited evidence related to self-management in PNCs. ${ }^{11}$ However, evidence on the implementation of SMS for many other patient populations was identified and may be able to provide useful insights where conditionspecific literature is sparse. One challenge for the review team will be in trying to decide to what extent knowledge gained from other settings may be transferable to the context of supporting people with PNCs with the challenges discussed above. Significant stakeholder involvement in the review process should help to ensure the relevance to the population of interest.

\section{Context of the review}

The review is part of a larger planned project which forms the basis of a PhD for FD. The programme theories generated during the review process will be used to design a theory-based training intervention. Use of realist reviews for this purpose has been recommended ${ }^{23}$ and applied in other settings. ${ }^{24}$

\section{Current stage of review work}

The iterative nature of realist reviews means it is difficult to prespecify the direction of the review before significant work has already been undertaken to identify and prioritise areas of focus. The authors have attempted to strike a balance in producing this protocol at a point when the review has progressed sufficiently to be able to provide useful detail but not so far into the process to make this an entirely retrospective account. The accompanying online supplementary file indicates the steps in the review process completed at the time of writing and those still anticipated. Initial searching, data extraction and synthesis have all started, with further searching, extraction and synthesis planned. For ease of reading the initial search process is described retrospectively, and the subsequent searches, data extraction and synthesis are described prospectively. Our aim in publishing the protocol at this stage is to add transparency to the synthesis process, especially since the method is open to interpretation.

The synthesis is being undertaken in parallel with two other pieces of work. The iterative nature of the review will mean that learning collectively from these work streams can usefully inform the direction of further searches and the refinement of the programme theories. An online survey of health professionals working with people with an exemplar PNC mulitple sclerosis (MS) was distributed in April to May 2016 with the aim of getting a snapshot of current practice, future training interests and important barriers in relation to the provision of SMS. Although the primary purpose of the survey data was to help prioritise specific intervention content for the later phase of the work, the data relating to barriers may helpfully inform programme theory development in the synthesis. Interviews with a small group of key informants with experience of training health professionals in skills relating to SMS are planned for October 2016. We will use a convenience sample of contacts made by the stakeholder group from a range of different training backgrounds. These interviews will allow the early developing programme theories from the synthesis to be discussed with the participants and subsequently further refined. ${ }^{25} \mathrm{~A}$ clear audit trail will be maintained so that the sources of programme theories remain transparent and these will be clearly reported on. 


\section{Planned review strategy}

The planned review strategy was registered on the PROSPERO database (CRD42016035596). The review process will follow the five stages of realist review described by Pawson et $a:^{26}$ clarification of scope, searching for evidence, appraising evidence and extracting data, synthesis, and dissemination.

\section{Clarification of scope}

A period of reading around the subject was undertaken by the first author (FD) which allowed key recurring themes from the wider literature about SMS to be identified. In the literature relating to training health professionals in SMS, specific SMS skills (and confidence in their use), perceptions of workplace fit and belief in the concept of SMS itself all appeared to be influential factors. Research exploring the implementation of SMS in practice identified issues that included patient-level barriers, the influence of health professional, local multidisciplinary team and wider organisational characteristics. These issues were discussed at an initial stakeholder group meeting in March 2016. Our stakeholder group includes the study authors who are academics from health (two of whom also work clinically as general practitioners), social sciences and education, with interests in SMS and/or postgraduate health professional training. Other members of the group were clinicians working with people with a PNC (MS specialist nurse and occupational therapist), service users with PNCs, a researcher working for a SMS training provider and third sector representation (MS trust). Unlike a traditional systematic review, key stakeholders are consulted throughout the review process from refining the focus of the review to challenging or validating emerging review findings. ${ }^{27}$ Informed by the group's discussion on priority areas, two key review questions were formulated, with the overarching aim of improving understanding of the circumstances in which health professionals could implement and sustain SMS. Therefore, the scope of the review was planned to include both professionals' experiences of receiving training in supporting self-management and their experiences of applying this training in clinical practice.

The initial review questions chosen were:

1. What is the influence of a shared concept of SMS within healthcare teams caring for people with PNCs and how can it be achieved?

The first question aimed to examine what professionals thought about SMS, and how this was influenced by training interventions and existing contextual factors (eg, professional role, previous experience and workplace factors).

2. What is known about how SMS can be successfully tailored for people with PNCs?

This question aimed to focus on how SMS might need to be provided differently for people with PNCs than for other conditions, and to examine whether training health professionals to adopt a more flexible or tailored approach was important.

Although dementia could be classified as a PNC, people with dementia are usually managed by a different healthcare team (old age psychiatry) to people with other PNCs, so for the purpose of this review we have not included dementia within our definition of PNCs. ${ }^{28}$

\section{Search strategy}

Iterative searches were planned in line with the realist methodology. The overlap in the searching, extraction and synthesis processes is illustrated by the flow chart in online supplementary file S1. Our initial search strategy, designed with input from an information specialist used three search threads in combination: health professional terms, self-management terms and PNC terms (both relevant $\mathrm{MeSH}$ headings and free-text terms; see online supplementary file S2). Search terms relating to selfmanagement were informed by terms used in previous systematic reviews ${ }^{4} 1129$ and by terms which existing known papers were indexed under. ${ }^{30}$ At this stage the aim was to be as inclusive as possible. Therefore, terms relating to goal setting and health coaching were included as these were seen to be important skills related to supporting self-management but which might not be indexed under the term self-management. Although we initially planned to include a fourth search thread of terms relating to education or training, after piloting the searches we noted that relevant papers relating to implementation of SMS interventions were not identified, so we removed this thread from the search.

The initial search was developed for MEDLINE via Ovid and then adapted for other databases (EMBASE, Cochrane Library, CINAHL, PEDro, ERIC and PsycInfo). The search was limited to English language papers (due to resource constraints) and to papers published in the past 20 years (as the concept of SMS is relatively recent). Following a particularly high recall from a search engine previously found to have a low specificity in relation to this topic (EMBASE), ${ }^{11}$ additional limitations were placed on the search to ensure only the most relevant papers were retained (non-Organisation for Economic Co-operation and Development (OECD) countries, children, palliative care and diagnosis-related studies were excluded). Details of the search dates and terms used are provided in online supplementary file S2. Initial searches were performed in April to May 2016.

Going forward, a grey literature search for relevant websites and policy documents is planned. In addition, forward and backward citation tracking of key papers will be used together with hand searching of relevant journals. Key papers already known to the authors, and identified through initial scoping exercises will also be eligible for inclusion, as will any recommended by members of the stakeholder group. A clear audit trail of the source of included papers will be maintained. The need for and direction of further iterative searching will be informed by the findings of the ongoing synthesis, 
stakeholder advisory group and key informant interviews as described below.

\section{Data extraction}

Titles will initially be screened for basic relevance by FD. Any titles that are obviously irrelevant will be excluded at this stage. Studies will be excluded if they focus predominantly on: paediatric patients, carers or families, nursing homes/managed care settings, diagnostic or end-of-life period, epidemiology, imaging or testing, measurement instruments, and specific treatments or devices.

An abstract screening tool developed by FD and tested in collaboration with FW will then be used to screen the remaining abstracts (see online supplementary file S3 for further detail). The tool will rank papers 1-4 based on their perceived likely relevance to either of the review questions. In brief, the highest ranked abstracts will be those that both related to a PNC and to health professionals' experiences of training in or implementation of SMS. Papers not specific to PNCs will be ranked lower, and those where professional involvement in SMS is unclear will be ranked as least likely to be relevant. Although the tool provides basic guidance on the likely relevance of papers for inclusion, author expertise and judgement will also be applied here to ensure that the tool is flexible enough to ensure potentially highly relevant papers are not deprioritised because they do not meet the predefined criteria. This application of researcher judgement is a key element of the realist approach to literature review which differs significantly from traditional systematic review. ${ }^{20}$ The full text of all papers ranked of the highest relevance will be sourced and assessed for potential inclusion. Full-text screening of the lower ranked abstracts will be undertaken selectively once data extraction from the initial papers provides further direction. If data saturation for some areas of the review is achieved early in the review process then it is anticipated that including data from these studies is unlikely to provide additional new information. Decisions about saturation will be made collaboratively through discussion among the authors.

Realist reviewers do not generally rely on traditional quality assessment tools, but instead make judgements on each piece of included evidence based on relevance and rigour. ${ }^{20}$ At the full-text screening stage, prior to data extraction, the researcher will decide whether the paper can provide information relevant to the research questions. Reasons for exclusion on the basis of relevance will be recorded. The assessment of rigour will be an ongoing process in the data extraction and synthesis phases. The researcher will critically reflect on all evidence during this phase with the aim of safeguarding the inferences made on the basis of individual extracts by ensuring that they are used appropriately. ${ }^{20}$

A core set of descriptors for each study will be collected including identifiers (author, title, year), type of data (primary evidence, review, opinion piece), patient group details, staff group details, brief description of intervention, relationship with other studies included in the review and setting (country and healthcare setting). Data relevant to the research questions will be extracted in the form of explanatory accounts configured as 'IfThen' statements. For example: 'If self-management is not valued by colleagues Then staff will feel discouraged from applying training in practice'. This approach was successfully used by another realist synthesis project which aimed to inform future training design. ${ }^{24}$ Extracting data as If-Then configurations rather than as context-mechanism-outcome configurations (the standard expression of realist programme theories) has the advantages of being an accessible way of starting to extract data with a 'realist lens', and providing a practical way for partial knowledge to accrue through the review process. ${ }^{24}$ A single 'If-Then' configuration may not contain each element of context, mechanism and outcome but may still be informative for the synthesis. When explanatory accounts derived from one data source are recognised to recur in another, this will be noted. The principles of meta-ethnography ${ }^{31}$ have been applied during realist synthesis in order to provide clear evidence of the type of data that is used to support the theories developed. ${ }^{32}$ We will follow this model so that during the extraction process data will be labelled as first order (direct from participants), second order (from study authors' interpretation) or third order (from synthesisers' interpretation of participants and authors' statements).

\section{Data synthesis}

We will take a similar approach to that described by Pearson $e t a l^{24}$ to consolidating our initial explanatory accounts into more refined programme theories. The data synthesis process will begin while data extraction is still ongoing and be facilitated by regular discussion between the review team members. Initially the first author will group together apparently linked explanatory accounts. NVivo V.10 (QSR International) will then be used to facilitate movement between the explanatory accounts and original data. A 'node' will be created for each group of linked accounts and original data that was used to derive the constituent explanatory accounts will be coded under this node. This will allow the reviewers to look back at the original data when generating a consolidated account, to help ensure that the consolidated account continues to accurately reflect the source material. The consolidation process, which will result in refined explanatory accounts, will be carried out in conjunction with a second author (FW). In addition, throughout this process (once early in the consolidation process and once towards the end to confirm the refined context-mechanism-outcome configurations) two further stakeholder meetings will be held. The stakeholders will have an important role in ensuring that the researchers' interpretations of the literature are seen as both relevant and important by professionals. The 
stakeholder group will also help to prioritise which of the explanatory accounts are seen as crucially important to continue to pursue and which may be of less immediate relevance. If 'priority' programme theories are felt to be described in insufficient detail by the literature identified in the initial searches, supplementary targeted searches of the academic and grey literature will be performed.

Key informant interviews are also planned. Individuals with experience of training health professionals using a variety of different approaches, all of which may relate to supporting self-management in some way, will be recruited (4-6 participants anticipated). These interviews will act as another check of the relevance of the theories developed. Trainers may also be able to help fill in the gaps not fully explored within the literature by reflecting on their own experiences, and to indicate whether any important areas have not yet been addressed.

During the later stages of the review, once the programme theories are relatively refined, existing middle range theories ${ }^{33}$ which could help to further our understanding of the programme theories will be sought. There are already a number of candidate middle-range theories known to the authors thought to be potentially relevant to the review (eg, diffusion of innovations and normalisation process theory). ${ }^{34} 35$ These known theories will be considered along with any substantive theories used within the included papers to explain their findings. If none of these theories prove to be a good explanatory fit, targeted searching for theory will be undertaken. ${ }^{36}$

\section{ETHICS AND DISSEMINATION}

Ethical approval is not required for the literature synthesis. However, ethical approval has been obtained for the online survey and supplementary interview data via Cardiff University School of Medicine Research Ethics Committee.

The RAMESES publication standards for realist synthesis have been consulted during the planning of the review and will be followed for future publication. ${ }^{37} \mathrm{We}$ will publish the synthesis in a peer-reviewed journal and make the findings available to relevant interested bodies including third sector organisations. We also aim for the theories to be useful to those designing training in SMS for health professionals, to help to identify what may be likely to work and where.

Owing to the relatively limited data expected to be available that are specific to the clinical area (PNCs) and the intervention (improving SMS provision by health professionals), we recognise that some of the theories developed during the synthesis may be partially or weakly supported. We will be fully transparent about the level of evidence available to support each theory developed to allow readers to draw their own conclusions about the relevance of the developed theories to their own contexts of interest.
Acknowledgements The authors would like to thank the members of the stakeholder group for their time and contributions to the study. The authors also thank Mala Mann for assistance with developing the initial search strategies.

Contributors FD planned the synthesis approach with input from $A B, A E, C W$ and FW. All authors participated in the initial stakeholder event to define the direction of the review. FD prepared the first draft of the protocol which was reviewed and critically revised by the other authors.

Funding The realist synthesis has been supported by a grant from the Royal College of General Practitioners Scientific Foundation Board (SFB 2015-18).

\section{Competing interests None declared.}

Provenance and peer review Not commissioned; externally peer reviewed.

Open Access This is an Open Access article distributed in accordance with the Creative Commons Attribution Non Commercial (CC BY-NC 4.0) license, which permits others to distribute, remix, adapt, build upon this work noncommercially, and license their derivative works on different terms, provided the original work is properly cited and the use is non-commercial. See: http:// creativecommons.org/licenses/by-nc/4.0/

\section{REFERENCES}

1. Lorig KR, Holman HR: Self-management education: history, definition, outcomes, and mechanisms. Ann Behav Med 2003;26:1-7.

2. Corbin J, Strauss A: Unending Work and Care: Managing Chronic Illness at Home. San Francisco: Jossey-Bass, 1988.

3. Morgan HM, Entwistle VA, Cribb A, et al. We need to talk about purpose: a critical interpretive synthesis of health and social care professionals' approaches to self-management support for people with long-term conditions. Health Expect 2016; doi:10.1111/ hex.12453.

4. Boger E, Ellis J, Latter S, et al. Self-management and self-management support outcomes: a systematic review and mixed research synthesis of stakeholder views. PLOS ONE 2015;10:e0130990.

5. Rogers A, Kennedy A, Bower P, et al. The United Kingdom Expert Patients Programme: results and implications from a national evaluation. Med J Aust 2008;189:S21.

6. de Silva D. Evidence: helping people help themselves. London: The Health Foundation, 2011.

7. Fraser R, Ehde D, Amtmann D, et al. Self-management for people with multiple sclerosis: report from the first international consensus conference, November 15, 2010. Int J MS Care 2013;15:99-106.

8. Plow MA, Finlayson M, Rezac M. A scoping review of self-management interventions for adults with multiple sclerosis. PM R 2011:3:251-62.

9. Barlow J, Edwards R, Turner A. The experience of attending a lay-led, chronic disease self-management programme from the perspective of participants with multiple sclerosis. Psychol Health 2009;24:1167-80

10. Hibbard JH, Gilburt H. Supporting people to manage their health: an introduction to patient activation. London: King's Fund, 2014

11. Taylor SJ, Pinnock H, Epiphaniou E, et al. A rapid synthesis of the evidence on interventions supporting self-management for people with long-term conditions: PRISMS-Practical systematic Review of Self-Management Support for long-term conditions. Health Serv Deliv Res 2014;2:53.

12. Battersby M, Lawn S, Wells L, et al. An analysis of training and information options to support chronic disease prevention and self-management in primary health care. Adelaide: Flinders University, 2008.

13. Jones F, Gage H, Drummond A, et al. Feasibility study of an integrated stroke self-management programme: a cluster-randomised controlled trial. BMJ Open 2016;6:e008900.

14. Kennedy A, Rogers A, Bowen R, et al. Implementing, embedding and integrating self-management support tools for people with long-term conditions in primary care nursing: a qualitative study. Int J Nurs Stud 2014;51:1103-13.

15. McKenna S, Jones F, Glenfield P, et al. Bridges self-management program for people with stroke in the community: a feasibility randomized controlled trial. Int J Stroke 2015;10:697-704.

16. Ong BN, Rogers A, Kennedy A, et al. Behaviour change and social blinkers? The role of sociology in trials of self-management behaviour in chronic conditions. Sociol Health IIIn 2014;36:226-38. 
17. Davies F, Wood F, Brain K, et al. The transition to secondary progressive multiple sclerosis: an exploratory qualitative study of health professionals' experiences. Int J MS Care 2016;18:257-64.

18. Axelrod L, Gage H, Kaye J, et al. Workloads of Parkinson's specialist nurses: implications for implementing national service guidelines in England. J Clin Nurs 2010;19:3575-80.

19. Cervero RM, Gaines JK. The impact of CME on physician performance and patient health outcomes: an updated synthesis of systematic reviews. J Contin Educ Health Prof 2015;35:131-8.

20. Pawson R. Evidence-based policy: a realist perspective. London: SAGE Publications, 2006.

21. Craig $P$, Dieppe $P$, Macintyre $S$, et al. Developing and evaluating complex interventions: the new Medical Research Council guidance. BMJ 2008;337:a1655.

22. Panagioti M, Richardson G, Murray E, et al. Reducing Care Utilisation through Self-management Interventions (RECURSIVE): a systematic review and meta-analysis. Health Serv Deliv Res 2014;2:54.

23. Fletcher A, Jamal F, Moore G, et al. Realist complex intervention science: applying realist principles across all phases of the Medical Research Council framework for developing and evaluating complex interventions. Evaluation 2016;22:286-303.

24. Pearson M, Brand S, Quinn C, et al. Using realist review to inform intervention development: methodological illustration and conceptual platform for collaborative care in offender mental health. Implement Sci 2015;10:134.

25. Manzano A. The craft of interviewing in realist evaluation. Evaluation 2016;22:342-60.

26. Pawson R, Greenhalgh T, Harvey G, et al. Realist review-a new method of systematic review designed for complex policy interventions. J Health Serv Res Policy 2005;10(Suppl 1):21-34.
27. Rycroft-Malone J, McCormack B, Hutchinson AM, et al. Realist synthesis: illustrating the method for implementation research. Implement Sci 2012;7:33.

28. National Audit Office. Services for people with neurological conditions: progress review. London: National Audit Office, 2015.

29. Mudge S, Kayes N, McPherson K. Who is in control? Clinicians' view on their role in self-management approaches: a qualitative metasynthesis. BMJ Open 2015;5:e007413.

30. Yank V, Laurent D, Plant K, et al. Web-based self-management support training for health professionals: a pilot study. Patient Educ Couns 2013;90:29-37.

31. Atkins $\mathrm{S}$, Lewin $\mathrm{S}$, Smith $\mathrm{H}$, et al. Conducting a meta-ethnography of qualitative literature: lessons learnt. BMC Med Res Methodol 2008;8:1.

32. Punton M, Vogel I, Lloyd R. Reflections from a realist evaluation in progress: scaling Ladders and Stitching Theory. Centre for Development Impact Practice Paper, 2016:18. https://opendocs.ids. ac.uk/opendocs/handle/123456789/11254

33. Merton R. On theoretical sociology: five essays, old and new. New York: The Free Press, 1967.

34. Rogers EM. Diffusion of innovations. 5th edn. New York: Free Press, 2003.

35. May C, Finch T. Implementing, embedding, and integrating practices: an outline of normalization process theory. Sociology 2009;43:535-54.

36. Booth A, Carroll C. Systematic searching for theory to inform systematic reviews: is it feasible? Is it desirable? Health Info Libr J 2015;32:220-35.

37. Wong G, Greenhalgh T, Westhorp G, et al. RAMESES publication standards: realist syntheses. BMC Med 2013;11:21. 